\title{
EQUATIONS OF MOTION FOR FIELD OPERATORS AND ENERGY SPECTRUM FOR SUPERFLUID ANISOTROPIC FERMI SYSTEMS ("p" PAIRING)
}

\author{
Z. M. Galasiewicz \\ Wroclaw University of Technology, Institute of Physics, \\ Wybrzeże Wyspiańskiego 27, 50-370 Wroclaw, Poland
}

(Received June 21, 1999)

\begin{abstract}
The investigation has been based on Hamiltonian quadratic in Fermi amplitudes or linear in operators representing amplitude and phase of condensate of pair (parallel spins). The latter represents the energy of a "spin" $1 / 2 \hat{\mathbf{L}}$ in some effective field. Simple variational procedure gives for anisotropic ABM and "polar" phases single particle energy $\boldsymbol{\Omega}$ with a suitable form of energy gap. Amplitude and phase oscillations are connected with frequencies $\boldsymbol{\omega}=0, \pm 2 \Omega$. The first one represents Goldstone-Anderson mode. "Spins" dependence on time $\left(\hat{\mathbf{L}}^{(i)}(t)=R_{i j}(t) \hat{\mathbf{L}}^{(j)}(0)\right)$ is given by rotation matrix $R_{i j}(t)$ used in $\mathrm{He}^{3}-\mathrm{B}$ theory. Here $R_{i j}$ describes rotation of a "spin" around the effective field.
\end{abstract}

Key words: superfluidity, anisotropic phases, "p" pairing, Casimir operator.

PACS number: 67.57.-z, 05.30.Fk

\section{THE EFFECTIVE HAMILTONIAN}

In [1] we considered systems of fermions described in terms of model Hamiltonian which was a quadratic form in Fermi field operators.

Interaction between particles led to "s" pairing giving a simple model of the superconductor with the energy spectrum having the isotropic gap.

Now we are interested in superfluid Fermi systems with the anisotropic energy gap. This is connected with interaction between particles which leads to "p" pairing.

We wish to consider a simple model which has some properties similar to superfluid $\mathrm{He}-3$ in anisotropic phases named now: polar or "one-dimensional" phase and A.B.M. phase (see e.g. [2]).

First model ("polar") of an anisotropic superfluid Fermi system has been investigated in [3]. It was described by the Hamiltonian:

$$
\begin{aligned}
\hat{H} & =\sum_{p, \sigma} \varepsilon(p) a_{p \sigma}^{+} a_{p \sigma} \\
& +\frac{1}{2 V} \sum_{p, p^{\prime}, \sigma} \sum_{n} J_{n}\left(p, p^{\prime}\right) P_{n}(\cos \gamma) a_{p \sigma}^{+} a_{-p \sigma}^{+} a_{-p^{\prime} \sigma} a_{p^{\prime} \sigma}
\end{aligned}
$$

where $P_{0}=1, P_{1}(\cos \gamma)=\cos \gamma=\cos v \cos v^{\prime}+$ $\cos \varphi \sin v \sin v^{\prime}$ denotes the angle between $p$ and $p^{\prime}$ vectors, $v$ and $v^{\prime}$ denote angles between the polar axis and the vectors $p$ and $p^{\prime}$ respectively, $\varphi$ denotes the angle around the polar axis.

A possible simple form of the interaction consistent with "p" pairing is:

$$
\sum P_{n} \simeq J_{1}\left(p_{F}, p_{F}\right) P_{1}(\cos \gamma)=J_{1}\left(p_{F}\right) \cos \gamma
$$

(in [1] only the zero term has been retained i.e.: $\sum_{n} P_{n} \simeq$ $J_{0}\left(p_{F}\right)$ ). Instead of (1.1) we wish to consider appropriate model Hamiltonian, which like in [1] will be a quadratic form in Fermi amplitudes. We assume:

$$
\begin{aligned}
\hat{H}_{\mathrm{red}} & =\sum_{p, \sigma} \varepsilon(p) a_{p \sigma}^{+} a_{p \sigma} \\
& -\frac{1}{2 V} \sum_{p, p^{\prime}, \sigma} J_{1}\left(p_{F}\right) \cos \gamma\left\{\left\langle a_{-p \sigma}^{+} a_{p \sigma}^{+}\right\rangle a_{-p^{\prime} \sigma} a_{p^{\prime} \sigma}\right. \\
& \left.+\left\langle a_{p \sigma} a_{-p \sigma}\right\rangle a_{p^{\prime} \sigma}^{+} a_{-p^{\prime} \sigma}^{+}\right\}+ \text {const. }
\end{aligned}
$$

Here we have $\varepsilon(p)=E(p)-\lambda, E(p)$ means single particle energy spectrum and $\lambda$ chemical potential, $\langle\ldots\rangle$ denotes ground state averaging.

We perform the canonical transformation:

$$
\begin{gathered}
a_{p \sigma}=u(p) \alpha_{p \sigma}-v(p, \sigma) \alpha_{-p \sigma}^{+}, \\
a_{-p \sigma}=u(p) \alpha_{-p \sigma}+v(p, \sigma) \alpha_{p \sigma}^{+}
\end{gathered}
$$

For the coefficients $\{u, v\}$ which can be complex, we have:

$$
\begin{aligned}
& |u(p)|^{2}+|v(p)|^{2}=1 \\
& v(p, \sigma)=-v(-p, \sigma) .
\end{aligned}
$$

For further considerations it is convenient to introduce new functions $F, \phi, \phi^{*}$ (see e.g. [4]):

$$
\begin{aligned}
& F(p)=|v(p, \sigma)|^{2}=|v(p)|^{2} \\
& \phi(p, \sigma)=u(p) v(p, \sigma)=-\phi(-p) .
\end{aligned}
$$


They are connected by an equation of the type (1.5):

$$
F^{2}-F+\phi \phi^{*}=0
$$

In terms of $\alpha, \alpha^{+}$amplitudes Hamiltonian (1.3) has the form:

$$
\begin{aligned}
\hat{H}_{\mathrm{red}} & =\left\langle\hat{H}_{0}\right\rangle+\sum_{p, \sigma} \Omega(p) \alpha_{p \sigma}^{+} \alpha_{p \sigma} \\
& +\sum_{p, \sigma}\left(R_{p \sigma} \alpha_{-p \sigma}^{+} \alpha_{p \sigma}^{+}+R_{p \sigma}^{*} \alpha_{p \sigma} \alpha_{-p \sigma}\right)
\end{aligned}
$$

where:

$$
\begin{aligned}
&\left\langle\hat{H}_{0}\right\rangle=2 \sum_{p} \varepsilon(p) F(p) \\
&+\sum_{p}\left(\Delta^{*}(p) \phi(p)+\Delta(p) \phi^{*}(p)\right), \\
& \Omega(p)=\varepsilon(p)(1-F(p)) \\
&+\Delta^{*}(p) \phi(p)+\Delta(p) \phi^{*}(p), \\
& R_{p \sigma}=2 \varepsilon(p) \phi^{*}(p)-\Delta^{*}(p) u^{2}(p)+\Delta(p) v^{*^{2}}(p), \\
& R_{p \sigma}^{*}=2 \varepsilon(p) \phi(p)-\Delta(p) u^{*^{2}}(p)+\Delta^{*}(p) v^{2}(p)
\end{aligned}
$$

From (1.15) it follows:

$$
\gamma=-\frac{\Delta}{\phi}=-\frac{\Delta^{*}}{\phi^{*}}
$$

and we have simple equations equivalent to (1.13)

$$
\begin{aligned}
& 2 \varepsilon \phi-\Delta(2 F-1)=0, \\
& 2 \varepsilon \phi^{*}-\Delta^{*}(2 F-1)=0 .
\end{aligned}
$$

and

$$
\begin{aligned}
\Delta(p, \sigma) & =-\frac{1}{2 V} \sum_{p^{\prime}} J_{1}\left(p_{F}\right)(\cos \gamma)\left\langle a_{p^{\prime} \sigma} a_{-p^{\prime} \sigma}\right\rangle \\
\Delta^{*}(p, \sigma) & =-\frac{1}{2 V} \sum_{p^{\prime}} J_{1}\left(p_{F}\right)(\cos \gamma)\left\langle a_{-p^{\prime} \sigma}^{+} a_{-p^{\prime} \sigma}^{+}\right\rangle
\end{aligned}
$$

One can check that when $\langle\ldots\rangle$ means averaging in vacuum of $\alpha, \alpha^{+}$quasiparticles, we have:

$$
\left\langle a_{p^{\prime} \sigma} a_{-p^{\prime} \sigma}\right\rangle=\phi\left(p^{\prime}\right)
$$

When we put

$$
R_{p \sigma}=R_{p \sigma}^{*}=0
$$

(the so called equation of compensation of dangerous diagrams) Eqs. (1.5) (or (1.7)) and (1.13) will give solutions for $F, \phi$, describing superfluid phases of $\mathrm{He}^{-} 3$. Unfortunately it is not easy to express $R_{p \sigma}$ in terms of $F, \phi$. We can find equations equivalent to (1.13) from variational procedure. We can perform variation of $\left\langle\hat{H}_{0}\right\rangle$, with respect to $F, \phi, \phi^{*}$ considering (1.7) by introducing a Lagrange multiplier $\gamma(p)$.

and have

$$
\begin{aligned}
& \frac{\delta W}{\delta F}=\varepsilon+\gamma(2 F-1)=0 \\
& \frac{\delta W}{\delta \phi}=\Delta^{*}+\gamma \phi^{*}=0 \\
& \frac{\delta W}{\delta \phi^{*}}=\Delta+\gamma \phi=0 .
\end{aligned}
$$

Eqs. (1.7), (1.17) give finally

$$
\begin{aligned}
& \phi=\frac{1}{2} \Delta /\left(\varepsilon^{2}+|\Delta|^{2}\right)^{1 / 2}, \\
& F=\frac{1}{2}\left[1-\varepsilon /\left(\varepsilon^{2}+|\Delta|^{2}\right)^{1 / 2}\right], \\
& \Omega=\left(\varepsilon^{2}+|\Delta|^{2}\right)^{1 / 2}, \quad \lambda=2 \Omega .
\end{aligned}
$$

\section{TIME DEPENDENCE OF THE} CONDENSATE AMPLITUDE AND PHASE. AMPLITUDE AND PHASE MODES

With the help of (1.11) Hamiltonian (1.3) has now the form

$$
\begin{aligned}
\hat{H}_{\mathrm{red}}= & \sum_{p, \sigma} \varepsilon(p)\left[a_{p \sigma}^{+} a_{p \sigma}+a_{-p \sigma}^{+} a_{-p \sigma}\right] \\
+ & \sum_{p, \sigma}\left[\Delta(p, \sigma) a_{p \sigma}^{+} a_{-p \sigma}^{+}+\Delta^{*}(p, \sigma) a_{-p \sigma} a_{p \sigma}\right] \\
& \Delta(p, \sigma)=\Delta(p)=-\Delta(-p) \\
& \Delta(p)=\Delta_{1}(p)+i \Delta_{2}(p)
\end{aligned}
$$

Using (2.1) we can get the following equations of motion for operators $a_{p \sigma}, a_{p \sigma}^{+}$ 


$$
\begin{aligned}
i \partial_{t} a_{p \sigma} & =\varepsilon(p) a_{p \sigma}+\Delta(p) a_{-p \sigma}^{+} \\
-i \partial_{t} a_{-p \sigma}^{+} & =\varepsilon(p) a_{-p \sigma}^{+}-\Delta^{*}(p) a_{p \sigma} .
\end{aligned}
$$

Now we introduce creation and annihilation operators of fermion pairs when "mates" have opposite momenta but parallel spins

$$
\begin{gathered}
\hat{\phi}_{p}(\sigma)=a_{-p \sigma} a_{p \sigma}=-\hat{\phi}_{-p}(\sigma), \\
\hat{\phi}_{-p}(\sigma)=a_{-p \sigma}^{+} a_{p \sigma}^{+}=-\hat{\phi}_{p}(\sigma) .
\end{gathered}
$$

We can define, with the help of $\hat{\phi}_{p}(\sigma), \hat{\phi}_{-p}^{+}(\sigma)$ the operators which can be connected with an expression for superfluid phase $\left(B^{(1)}\right)$ and density of pairs condensate $\left(B^{(2)}\right)[5]$, (see also [6]). Namely

$$
\begin{aligned}
& \hat{B}_{p}^{(1)}(\sigma)=\hat{\phi}_{p}(\sigma)+\hat{\phi}_{-p}^{+}(\sigma)=\hat{B}_{p}^{(1)^{+}}(\sigma), \\
& \hat{B}_{p}^{(2)}(\sigma)=i\left(\hat{\phi}_{p}(\sigma)-\hat{\phi}_{-p}^{+}(\sigma)\right)=\hat{B}_{p}^{(2)^{+}}(\sigma) .
\end{aligned}
$$

Operators $B^{(1)}, B^{(2)}$ depend on $p$ (and not on $p \pm q / 2$ ), so they represent only a part of the expression for amplitude and phase operators. Definition (2.3) of $\hat{\phi}, \hat{\phi}^{+}$leads to the following properties [7]

$$
\hat{\phi}^{2}=0, \quad \hat{\phi} \hat{\phi}^{+} \hat{\phi}=\hat{\phi} .
$$

They give us a possibility to express Hamiltonian (2.1) in terms of operators similar to $B^{(i)}$ but isomorphic to Pauli matrices. We introduce

$$
\begin{aligned}
\hat{L}_{p}^{(1)}(\sigma) & =\hat{B}_{p}^{(1)}(\sigma)=\hat{\phi}_{-p}^{+}(\sigma)+\hat{\phi}_{p}(\sigma), \\
\hat{L}_{p}^{(2)}(\sigma) & =-\hat{B}_{p}^{(2)}(\sigma)=i\left(\hat{\phi}_{-p}^{+}(\sigma)-\hat{\phi}_{p}(\sigma)\right), \\
\hat{L}_{p}^{(3)}(\sigma) & =\hat{\phi}_{p}(\sigma) \hat{\phi}_{-p}^{+}(\sigma)-\hat{\phi}_{-p}^{+}(\sigma) \hat{\phi}_{p}(\sigma) \\
& =1-n_{p \sigma}-n_{-p \sigma}, \\
\hat{J}_{p}(\sigma) & =\hat{\phi}_{-p}^{+}(\sigma) \hat{\phi}_{p}(\sigma)+\hat{\phi}_{p}(\sigma) \hat{\phi}_{-p}^{+}(\sigma) \\
& =\left(\hat{J}_{p}(\sigma)\right)^{2}=\hat{L}_{p}^{(3)}(\sigma)+2 n_{p \sigma} n_{-p \sigma},
\end{aligned}
$$

$L^{(3)}$ enters in a natural way to the commutator of $B^{(1),(2)}$ (or $L^{(1),(2)}$ ) with the Hamiltonian, $\hat{J}$ plays the role of unity.

Operators (2.6) have the following properties

$$
\begin{aligned}
& {\left[\hat{L}_{p}^{(l)}, \hat{L}_{p}^{(j)}\right]=2 i \varepsilon_{l j k} \hat{L}_{p}^{(k)},} \\
& \hat{L}_{p}^{(j)} \hat{L}_{p}^{(k)}=i \varepsilon_{j k l} \hat{L}_{p}^{(l)}, \\
& \left(\hat{L}_{p}^{(i)}\right)^{2}=\hat{J}_{p},
\end{aligned}
$$

$$
\hat{L}_{p}^{(i)} \hat{J}_{p}=\hat{J}_{p} \hat{L}_{p}^{(i)}=\hat{L}_{p}^{(i)}, \quad i=1,2,3 .
$$

We mentioned earlier that $L^{(i)}, \hat{J}$ are isomorphic to Pauli matrices and unity matrix.

Introduction of $L^{(i)}$ into Hamiltonian (2.1) (we do not perform unitary transformation) gives

$$
\begin{aligned}
\hat{H}_{\mathrm{red}} & =\sum_{p, \sigma} \mathbf{h}(p) \hat{\mathbf{L}}_{p}(\sigma)=\sum_{p, \sigma} \Omega(p) \mathbf{n}(p) \hat{\mathbf{L}}_{p}(\sigma), \\
& =\sum_{p, \sigma} \hat{H}_{p}(\sigma) \\
\mathbf{h}(p) & =\left(\Delta_{1}(p), \Delta_{2}(p),-\varepsilon(p)\right) \\
|\mathbf{h}(p)| & =\Omega(p)=\sqrt{\varepsilon^{2}(p)+|\Delta(p)|^{2}}
\end{aligned}
$$

where $\mathbf{h}(p)$ can be considered as effective magnetic field acting on "spin" $(1 / 2) \hat{\mathbf{L}}$. We have here a situation simpler than in [8] where effective field is partly an operator and Hamiltonian is a quadratic form in spin operators.

In order to have information about the amplitude and phase oscillations let us find the time dependence of the components of vector $\hat{\mathbf{L}}$. Comparatively a simple form of the Hamiltonian (2.8) (linear form in $\hat{L}^{(i)}$ ) suggests to use the formula

$$
\begin{aligned}
\hat{\mathbf{L}}(t) & =\exp [-i \hat{H} t] \hat{\mathbf{L}}(0) \exp [i \hat{H} t] \\
& =\sum_{n=0}^{\infty} \frac{t^{n}}{n !}(-i)^{n}(\left.\underbrace{[\hat{H},[\hat{H}, \ldots[\hat{H}, \hat{L}]]]}_{n}\right|_{t=0}),
\end{aligned}
$$

where commutators represent suitable derivatives with respect to time. We find

$$
\begin{aligned}
& \frac{d^{2 n}}{d t^{2 n}} \hat{\mathbf{L}}(t)=(-1)^{n}(2 \Omega)^{2 n}\left[\hat{\mathbf{L}}(t)-\frac{\mathbf{h}(\mathbf{h} \cdot \hat{\mathbf{L}})}{\Omega^{2}}\right], \quad n \neq 0 \\
& \frac{d^{2 n+1}}{d t^{2 n+1}} \hat{\mathbf{L}}(t)=\frac{(-1)^{n}}{2 \Omega}(2 \Omega)^{2 n+1} \frac{d \hat{\mathbf{L}}(t)}{d t} .
\end{aligned}
$$

In a special case

$$
\frac{d^{3} \hat{\mathbf{L}}}{d t^{3}}+(2 \Omega)^{2} \frac{d \hat{\mathbf{L}}}{d t}=0
$$

The solution of (2.11) should be a linear combination of $\mathrm{e}^{i \omega t}$ with $\omega=0, \pm 2 \Omega$. Further considerations will give a more clear picture of this situation.

The commutation relations give us

$$
\frac{d \hat{\mathbf{L}}}{d t}=2 \mathbf{h} \times \hat{\mathbf{L}}
$$




\section{Z. M. GALASIEWICZ}

which is consistent with conservation of energy, namely

$$
\mathbf{h} \frac{d \hat{\mathbf{L}}}{d t}=0=\frac{d \hat{H}_{p}(\sigma)}{d t}
$$

When we put (2.12) into the second formula of (2.10) we see that all time derivatives depend linearly on $\hat{\mathbf{L}}$.

After use of (2.9) and (2.11) we have

$$
\begin{aligned}
\hat{\mathbf{L}}(t) & =(\hat{\mathbf{L}}(0) \cdot \mathbf{n}) \mathbf{n}+[\hat{\mathbf{L}}(0)-(\hat{\mathbf{L}}(0) \cdot \mathbf{n}) \mathbf{n}] \cos (2 \Omega t) \\
& +(\mathbf{n} \times \hat{\mathbf{L}}(0)) \sin (2 \Omega t) \\
\hat{L}^{(j)}(t) & =\left(\hat{L}^{(i)}(0) n_{i}\right) n_{j} \\
& +\left[\delta_{i j} \hat{L}^{(i)}(0)-\left(\hat{L}^{(i)}(0) n_{i}\right) n_{j}\right] \cos (2 \Omega t) \\
& +\varepsilon_{j k i} n_{k} \hat{L}^{(i)}(0) \sin (2 \Omega t)=R_{j i}[t] \hat{L}^{(i)}(0)
\end{aligned}
$$

where

$$
\begin{aligned}
R_{j i}[t] & =\cos (2 \Omega t)\left(\delta_{i j}-n_{j} n_{i}\right)+n_{j} n_{i} \\
& +\sin (2 \Omega t) \varepsilon_{j k l} n_{k}, \\
R_{j i}[0] & =\delta_{j i} .
\end{aligned}
$$

We see that

$$
\mathbf{n} \cdot \hat{\mathbf{L}}(t)=\mathbf{n} \cdot \hat{\mathbf{L}}(0)
$$

i.e. terms in (2.14) proportional to $\sin$ and cos are perpendicular to $\mathbf{n}$. Matrix $R_{j i}$ describes rotation around axis defined by unit vector $\mathbf{n} \| \mathbf{h}$ i.e. rotation in plane perpendicular to effective field. Such form of $R_{i j}$ is recently used very often in the theory of superfluid $\mathrm{He}^{3}-\mathrm{B}$.

From (2.11) and (2.14) follows that amplitude and phase oscillations are connected with frequencies $\omega=$ $0, \pm 2 \Omega$. The mode $\omega=0$ is a Goldstone mode or as it has been discussed more precisely in [9], the Anderson's [7], rotating ground state mode.

From (2.6) it follows that $L^{(3)}$ has eigenvalues

$$
L_{3}=-1,0,0,1 \text {, }
$$

and corresponding eigenstates

$$
a_{p \sigma}^{+} a_{-p \sigma}^{+}|0\rangle, \quad a_{p \sigma}^{+}|0\rangle, \quad a_{-p \sigma}^{+}|0\rangle, \quad|0\rangle
$$

We can introduce "spin" operators $\hat{\mathbf{S}}$

$$
\hat{\mathbf{S}}=\frac{1}{2} \hat{\mathbf{L}}
$$

Component $\hat{S}^{(3)}$ has eigenvalues

$$
m_{s}=-\frac{1}{2}, 0,0, \frac{1}{2}
$$

The Casimir operator can be written

$$
\hat{C}^{2}=\sum_{i}\left(\hat{S}^{(i)}\right)^{2}=\frac{3}{4} \hat{J}^{2}=\frac{3}{4} \hat{J}=l(l+1) I
$$

with $l=1 / 2,0,0,1 / 2$.

It is convenient to consider the subspace defined by condition $n_{p \sigma}=n_{-p \sigma}[8]$. In this case $l=1 / 2, m_{s}=$ $\pm 1 / 2$.

We can easy diagonalise Hamiltonian (2.8) rotating our system in such a way that $\mathbf{n} \rightarrow \mathbf{n}^{\prime}(0,0,1)$ i.e. $\mathbf{n} \hat{\mathbf{L}}=\mathbf{n}^{\prime} \hat{\mathbf{L}}^{\prime}=L^{(3)^{\prime}}$.

\section{THE ENERGY SPECTRUM}

After obtaining the formulae (1.11), (1.12) and (1.17) we shall couple them to the relation below, well known as the equation for the energy gap.

In our case it becomes

$$
\begin{aligned}
\Delta(p, \sigma) & =-\frac{1}{2 V} \sum_{p^{\prime}} J_{1}\left(p_{F}\right) P_{1}(\cos \gamma) \Delta\left(p^{\prime}, \sigma\right) /\left(\varepsilon^{2}(p)\right. \\
& \left.+|\Delta(p, \sigma)|^{2}\right)^{1 / 2}
\end{aligned}
$$

and suitable Eq. for $\Delta^{*}$. Now we shall study (3.1). For that reason we can rewrite (1.2) in the form of the polynomial series, where $P_{n}$ are the Legendre functions:

$$
\begin{aligned}
P_{1}(\hat{p} \cdot \hat{p}) & =P_{1}(\cos \gamma) \\
& =\frac{4 \pi}{3} \sum_{m /-1}^{m / 1} Y_{1 m}(v, \varphi) Y_{1 m}\left(v^{\prime}, \varphi^{\prime}\right)
\end{aligned}
$$

where

$$
\begin{aligned}
Y_{1 \pm 1}(v, \varphi) & =\left(\frac{3}{8 \pi}\right)^{1 / 2} \sin v(\cos \varphi \pm i \sin \varphi) \\
Y_{10}(v, \varphi) & =\left(\frac{3}{4 \pi}\right)^{1 / 2} \cos v
\end{aligned}
$$

Providing the calculations and discussion for (3.1) we shall replace the summation by the integration with the additional approximate assumption, that we are investigating the states near the Fermi momentum: 


$$
\frac{1}{V} \sum_{\mathbf{p}} \rightarrow \frac{1}{(2 \pi)^{3}} \int d^{3} \mathbf{p} \frac{1}{(2 \pi)^{3}} \int_{p \sim p_{F}} \int_{0}^{\pi} \int_{0}^{2 \pi}|p|^{2} d p \sin v d v d \varphi \simeq \frac{p_{F}^{2}}{(2 \pi)^{3}} \int_{p \sim p_{F}} \int_{0}^{\pi} \int_{0}^{2 \pi} d p d v d \varphi \sin v
$$

That way (taking into account $(3.4)$ and following: $\Delta\left(p_{F}, \hat{p}, \sigma\right)=\Delta(\hat{p}, \sigma)=\Delta(\hat{p})$, where $\left.\hat{\mathbf{p}}=\mathbf{p} /|\mathbf{p}|\right)$, the Eq. for the energy gap changes as below:

$$
\Delta(p)=\frac{J_{1}}{12 \pi^{2}} p_{F}^{2} \sum_{m /-1}^{m / 1} Y_{1 m}(v, \varphi) \int d p^{\prime} \int_{0}^{\pi} \int_{0}^{2 \pi} d v^{\prime} d \varphi^{\prime} \sin v^{\prime} \frac{Y_{1 m}^{*}\left(v^{\prime}, \varphi^{\prime}\right) \Delta\left(v^{\prime}, \varphi^{\prime}\right)}{\left\{\varepsilon^{2}(p)+\left|\Delta\left(v^{\prime}, \varphi^{\prime}\right)\right|^{2}\right\}^{1 / 2}}
$$

We can look for the solution of $\Delta(\hat{p})$ in the form of a linear combination:

$$
\Delta\left(v^{\prime}, \varphi^{\prime}\right)=\Delta_{m^{\prime}} Y_{1 m^{\prime}}\left(v^{\prime}, \varphi^{\prime}\right)
$$

On the right hand side of (3.5) we will obtain:

$$
\int Y_{1 m}^{*}\left(v^{\prime}, \varphi^{\prime}\right) Y_{1 m^{\prime}}\left(v^{\prime}, \varphi^{\prime}\right) d \varphi^{\prime}=\beta\left(v^{\prime}\right) \delta_{m m^{\prime}}
$$

Consideration of the "p" pairing is connected with distinguishing of some orbital axis 1 , for which $l_{z}=1$. In a coordinate system with $z$-axis parallel to 1 , unit momentum vector has the components:

$$
\hat{k}_{x}=\sin v \cos \varphi, \quad \hat{k}_{y}=\sin v \sin \varphi, \quad \hat{k}_{z}=\cos v
$$

and we see, that:

$$
Y_{1 \pm 1} \simeq \hat{k}_{x} \pm i \hat{k}_{y}, \quad Y_{10} \simeq \hat{k}_{z}
$$

We will discuss the most interesting solutions, leading to the anisotropic phases of superfluid Fermi systems. If we choose (3.6) in the form:

$$
\Delta=\Delta(v)=\Delta_{0} Y_{10}(v)=\Delta_{0} \cos v=\Delta_{0} \hat{k}_{z}
$$

$\left(m^{\prime}=0\right)$ we have the energy spectrum for a highly anisotropic "polar" phase:

$$
\Omega(p)=\left\{(E(p)-\lambda)^{2}+\Delta_{0}^{2} \cos ^{2} v\right\}^{1 / 2}
$$

considered first in 1960 by Galasiewicz (see [3], [2] and e.g. [11]).

In this case we shall say, that the system is "most superfluid" along the $z$-axis $\left(\Delta=\Delta_{0}\right)$.

Looking for the solution of (3.6) in the form:

$$
\begin{aligned}
\Delta & =\Delta(v, \varphi)=\Delta_{1} Y_{11}(v, \varphi)=\Delta_{1} \sin v \mathrm{e}^{i \varphi} \\
& =\Delta_{1}\left(\hat{k}_{x}+i \hat{k}_{y}\right) \\
& =\Delta_{1}(\sin v \cos \varphi+i \sin v \sin \varphi)
\end{aligned}
$$

we find the energy spectrum for anisotropic A.B.M. phase

$$
\Omega(p)=\left\{(E(p)-\lambda)^{2}+\Delta_{1}^{2} \sin ^{2} v\right\}^{1 / 2}
$$

considered first by Anderson and Morel (see e.g. [10]) in 1961.

In this case the spectrum describes the system "most superfluid" in the plane perpendicular to $\mathbf{1}$, which leads to the greater stability than in the "polar" phase. Then we have $\Delta=\Delta_{1}$. The expressions for $\Delta_{0}$ and $\Delta_{1}$ we can find from (3.5).

We see, that simple assumption putting in the Hamiltonian (2.1) $\Delta \sim Y_{00}$ leads to an isotropic BCS "s" dependence in (2.13). Assuming, that $\Delta \sim Y_{10}$ or $\Delta \sim Y_{11}$ gives us the anisotropic ("polar") type "p" dependence in (2.13) or A.B.M type in (2.10).
[1] Z. M. Galasiewicz, E. M. Ziemniak, Bull. Acad. Pol. Sci. 25, 715 (1977).

[2] A. Legget, Rev. Mod. Phys. 47, 331 (1975).

[3] Z. M. Galasiewicz, Dubna preprint (1959); Acta Phys. Pol. 19, 683 (1960).

[4] Z. M. Galasiewicz, Superconductivity and Quantum Fluids, (Pergamon Press, Oxford 1970).
[5] Z. M. Galasiewicz, Bull. Acad. Pol. Sci. 24, 353 (1976).

[6] A. Coniglio, M. Marinaro, Nuovo Cimento B 68, 249 (1967).

[7] P. W. Anderson, P. Morel, Phys. Rev. 123, 1911 (1961).

[8] H. Koppe, B. Mühlschlegel, Z. Phys. 151, 613 (1958).

[9] P. W. Anderson, Phys. Rev. 112, 1900 (1959).

[10] H. Stern, Phys. Rev. 147, 97 (1966). 
[11] H. W. Capel, in Ordering Phenomena in Condensed Matter Physics, XXVI Winter School of Theoretical
Physics, Karpacz, Poland 1990 (World Scientific, Singapore, 1991).

\title{
РІВНЯННЯ РУХУ ДЛЯ ПОЛЬОВИХ ОПЕРАТОРІВ ТА ЕНЕРІЕТИЧНИЙ СПЕКТР ДЛЯ НАДПЛИННИХ АНІЗОТРОПНИХ ФЕРМІ-СИСТЕМ ("р"СПАРЮВАННЯ)
}

\author{
3. М. Галасєвіч \\ Інститут фізики Вроилавсъкого технологічного університету, \\ вул. Вибжеже Виспяньскего, 27, Вроилав, 50-370, Польща
}

\begin{abstract}
Дослідження базується на гамільтоніяні ква,дратичному за амплітудами Фермі або лінійному за операторами, що зображають амплітуду та фазу конденсату пар (паралельних спінів). Останній зображає енергію "спіну" $1 / 2 \hat{\mathbf{L}}$ у деякому ефективному полі. Проста варіяпійна прощедура дає, для анізотропних АВМ та "полярноі” фаз одночастинкову енергію $\Omega$ з підхожою формою енергетичної шілини. Коливання амплітуди та фази пов'язані з частотами $\omega=0, \pm 2 \Omega$. Перша з них репрезентує моду Голдстоуна-Андерсона. Залежність "спіну" від часу $\left(\hat{\mathbf{L}}^{(i)}(t)=R_{i j}(t) \hat{\mathbf{L}}^{(j)}(0)\right)$ задана матрицею обертання $R_{i j}(t)$, яку використовують у Не ${ }^{3}-\mathrm{B}$ теорії. Тут $R_{i j}$ описує обертання "спіну" навколо ефективного поля.
\end{abstract}

